\title{
Microwave assisted synthesis of annelated benzosuberone as new penta-heterocyclic ring systems
}

\author{
Thoraya A. Farghaly and Sayed M. Riyadh* \\ Department of Chemistry, Faculty of Science, University of Cairo, Giza, Egypt \\ E-mail: riyadh1993@hotmail.com
}

\begin{abstract}
Derivatives of novel penta-heterocyclic ring systems, namely, 5,6,7,11-tetrahydro- $15 \mathrm{H}$ benzo[6',7']cyclohepta[1',2':4,5]pyrido[2,3- $d][1,2,4]$ triazolo[4,3- $a]$ pyrimidin-15-ones (9a-g) and 6,7-dihydrobenzo[6',7']cyclohepta[1',2':4,5]pyrido[2,3- $d][1,3]$ thiazolo[3,2-a]pyrimidin-15(5H)ones (11a-d) were easily prepared via reactions of 3-thioxo-2,3,4,7,8,9-hexahydro-1Hbenzo[6', $\left.7^{\prime}\right]$ cyclohepta[1',2':4,5]pyrido[2,3-d]pyrimidin-1-one (3) with hydrazonoyl halides and active methylene compounds, respectively. The foregoing reactions were carried out with conventional heating as well as pressurized microwave irradiation and a comparative study was employed.
\end{abstract}

Keywords: Penta-heterocyclic ring systems, hydrazonoyl halides, microwave irradiation, benzosuberone

\section{Introduction}

Benzocycloheptapyridines are active in inhibiting cholesterol biosynthesis and modulating blood serum lipids. ${ }^{1}$ The inhibition of the farnesyl protein transferase, a novel approach to antitumour therapy, is based on compounds such as piperazinyl substituted cycloheptapyridines. ${ }^{2}$ Various derivatives of 1,2,4-triazolopyrimidine have high affinity for binding with $A_{1}$ adenosine receptors, ${ }^{3}$ and the others can be used as calcium-channel-blocking vasodilators, antihypertensive, cardiovascular, anxiolytic activities and photographic materials. ${ }^{4-7}$ Thiazolo[3,2-a]pyrimidine derivatives have been ascertained as a new type of acetyl cholinesterase inhibitor for the treatment of Alzheimer disease. ${ }^{8}$ In this context, we have prepared novel pentacyclic ring systems incorporated benzo- cycloheptapyridines and both of 1,2,4-triazolopyrimidines and thiazolopyrimidines. Despite recent interest in microwave as 
energy source which enhances the reaction rates and improves the regioselectivity, ${ }^{9-12}$ the use of microwave in reactions of hydrazonoyl halides has received limited study. ${ }^{13}$

\section{Results and Discussion}

Various attention has been paid to the reactions of enaminones with amino heterocycles. ${ }^{14,15}$ To explore the utility of this, we investigated the reaction between 6-[(dimethylamino)methylene]6,7,8,9-tetrahydro-5H-benzo[7]annulen-5-one (1) and 6-amino-2-thioxo-2,3-dihydropyrimidin4(1H)-one (2) in glacial acetic acid [under reflux for 5 hours or pressurized microwave irradiation for $5 \mathrm{~min}$.]. This reaction produced tetra-heterocyclic ring system, namely, 3-thioxo2,3,4,7,8,9-hexahydro-1H-benzo[6',7']cyclohepta[1',2':4,5] pyrido[2,3-d]pyrimidin-1-one (3) or its isomeric structure (4) (Scheme 1). Mass, IR spectra and elemental analysis data of the isolated product were consistent with each of the isomeric structures 3 and $\mathbf{4}$ (Scheme 1), while, ${ }^{1} \mathrm{H} \mathrm{NMR}$ spectra revealed singlet signal at $\delta 8.23 \mathrm{ppm}$ assigned for pyridine-2H proton not pyridine-4H proton $^{16}$ which is consistent with the isomeric structure 3 . Furthermore, alternative synthesis of compound 3 was achieved via condensation of 6-amino-2-thioxo-2,3-dihydropyrimidin-4(1H)one (2) with dimethylformamide dimethylacetal (DMF-DMA) to give compound $5,{ }^{17}$ and treatment of the product 5 with benzosuberone to yield authentic product 3 (Scheme 1). In addition, literature reports explained that, the reaction of heterocyclic amine with enaminone proceeded firstly via nucleophilic attack of the amino group of the heterocyclic amine to the double bond of the enaminone with concurrent elimination of the dimethylamine rather than condensation of water molecule. ${ }^{18-20}$ On the basis of these findings, structure 4 was discarded and the isolated product from the studied reaction was assigned structure 3 (Scheme 1). It is worthily mentioned that compound 3 had been prepared by Remp et al ${ }^{21}$ via a different method.

Despite of numerous reports on the reactions of heterocyclic thiones with hydrazonoyl halides by conventional thermal heating, ${ }^{22}$ the utility of microwave in such reactions has received only limited study. Thus, heating of hydrazonoyl halides 6a-g with 3-thioxo-2,3,4,7,8,9hexahydro-1H-benzo[6',7']cyclohepta[1',2':4,5]pyrido[2,3-d]pyrimidin-1-one (3) in dioxane in the presence of triethylamine for 6-10 minutes in a pressurized microwave oven, or 20-24 hours by conventional thermal heating, afforded, in each case, one isolable product that was identified on the basis of its spectra (Ms, IR, ${ }^{1} \mathrm{H}$ NMR) and its elemental analysis data (see experimental). For example, whereas the IR spectrum of 9a shows only one CO band at $v 1699 \mathrm{~cm}^{-1}$, the IR spectra of 9b-g showed, in each case, two CO bands in the regions $1749-1700$ and $1702-1672$ $\mathrm{cm}^{-1}$. Their ${ }^{1} \mathrm{H}$ NMR spectra showed, in each case, one characteristic singlet signal at $\delta 8.18-$ $8.49 \mathrm{ppm}$ assignable to the pyridine $\mathrm{CH}$. The above results indicated that the products are novel penta-heterocyclic ring systems, namely, 5,6,7,11-tetrahydro-15H-benzo[6',7']cyclohepta$\left[1^{\prime}, 2^{\prime}: 4,5\right]$ pyrido[2,3- $\left.d\right][1,2,4]$ triazolo[4,3-a] pyrimidin-15-ones 9a-g as shown in Scheme 2. 


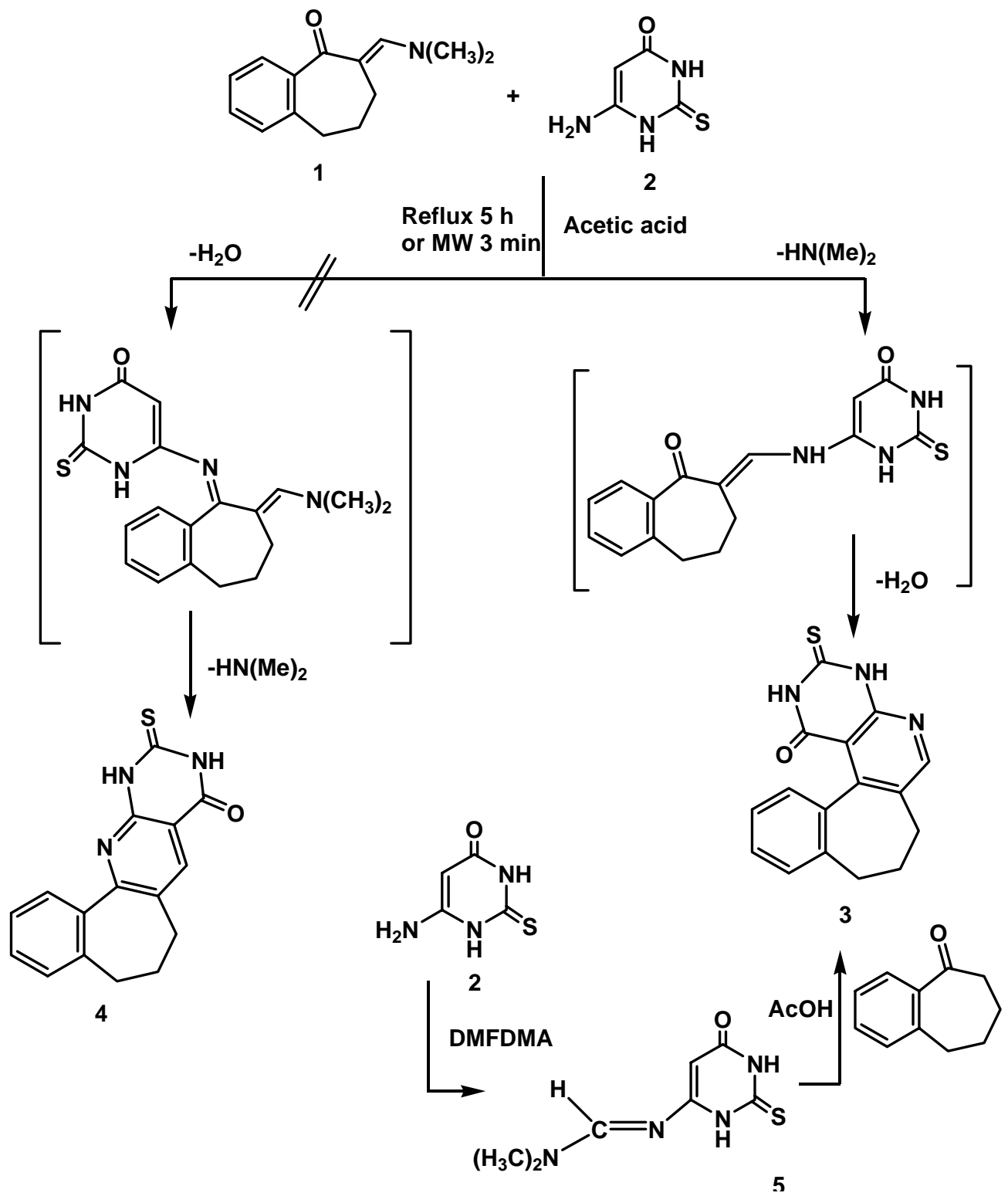

Scheme 1 

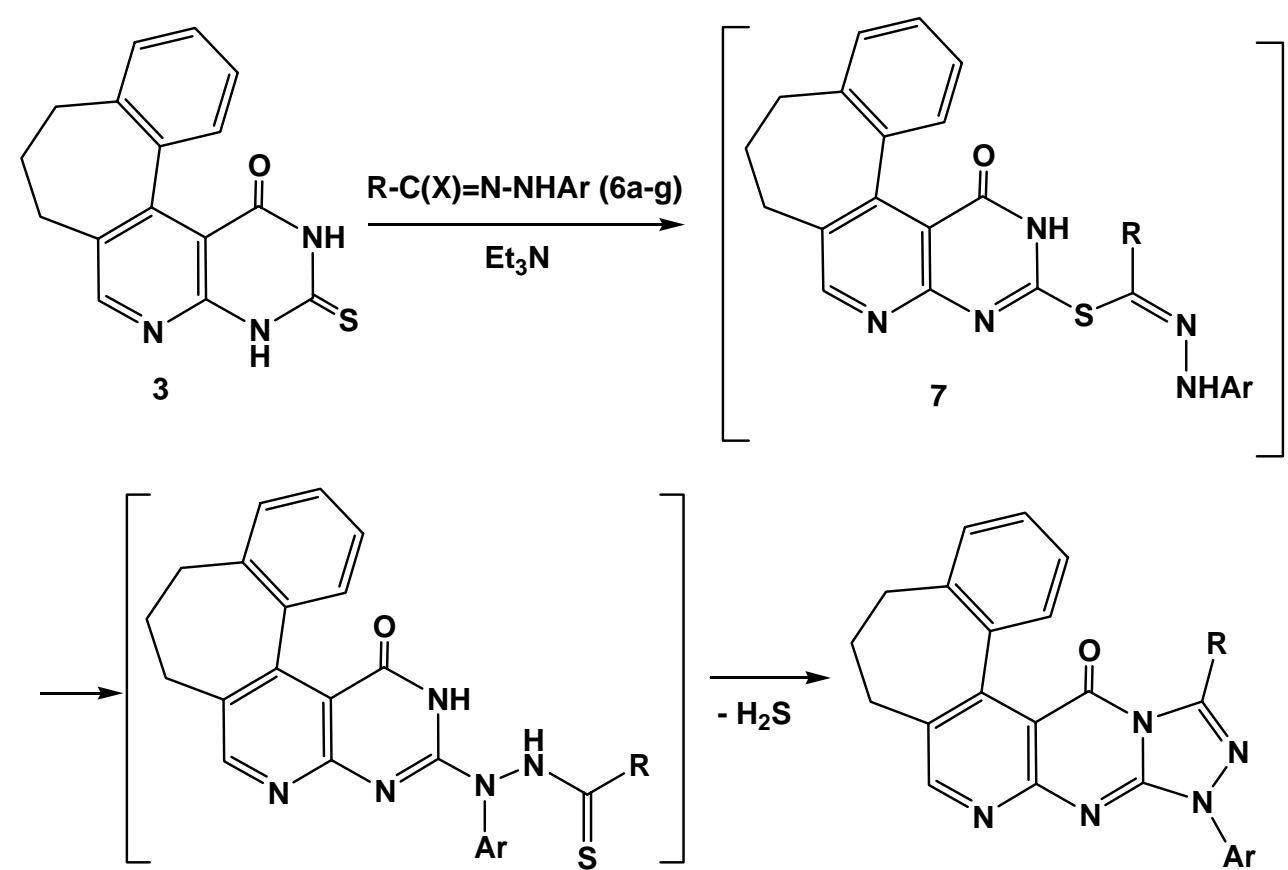

8<smiles>[R]c1nn([CH])c2nc3ncc4c(c3c(=O)n12)-c1ccccc1CCC4</smiles>

9a-g

\begin{tabular}{c|cc}
$\mathrm{X}=\mathrm{Cl}$ or $\mathrm{Br}$ & \\
$\begin{array}{c}\text { Compd. } \\
\text { no. }\end{array}$ & $\mathrm{R}$ & $\mathrm{Ar}$ \\
\hline $9 \mathrm{a}$ & $\mathrm{Ph}$ & $\mathrm{C}_{6} \mathrm{H}_{5}$ \\
9b & $\mathrm{CO}_{2} \mathrm{Et}$ & $\mathrm{C}_{6} \mathrm{H}_{5}$ \\
9c & $\mathrm{CH}_{3} \mathrm{CO}$ & $\mathrm{C}_{6} \mathrm{H}_{5}$ \\
9d & PhNHCO & $\mathrm{C}_{6} \mathrm{H}_{5}$ \\
9e & PhCO & $\mathrm{C}_{6} \mathrm{H}_{5}$ \\
9f & 2-Thienoyl & $4-\mathrm{ClC}_{6} \mathrm{H}_{4}$ \\
9g & 2-naphthoyl & $4-\mathrm{CH}_{3} \mathrm{C}_{6} \mathrm{H}_{4}$
\end{tabular}

Acetic acid

- $\mathrm{HN}\left(\mathrm{CH}_{3}\right)_{2}$

- $\mathrm{H}_{2} \mathrm{O}$

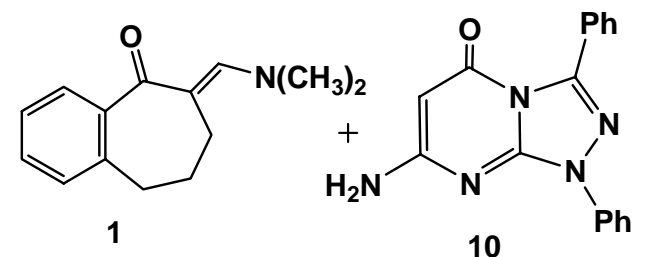

\section{Scheme 2}

As depicted in Scheme 2, the reaction proceeded through $S$-alkylation ${ }^{23}$ to give $S$-alkylated products 7 followed by Smiles rearrangement, ${ }^{24}$ affording intermediates 8 which eliminated hydrogen sulfide gas to give the desired products 9a-g. The assignment for the structure of the products and reaction mechanism can be manifested by alternate synthesis. Thus, treatment of 6[(dimethylamino)methylene]-6,7,8,9-tetrahydro-5H-benzo[7]annulen-5-one (1) with 7-amino1,3-diphenyl[1,2,4]triazolo[4,3-a]pyrimidin-5(1H)-one $(\mathbf{1 0})^{25}$ in acetic acid led to formation of product that proved to be identical in all respects ( $\mathrm{mp}$, mixed $\mathrm{mp}$ and IR) with compound 9a (Scheme 2). A comparative study of the yields of the products under pressurized microwave irradiation (method A) and conventional heating (method B) (Table 1) showed that the use of 
microwave irradiation substantially reduced the reaction times from hours scale to minutes scale and appreciably increased the yields.

Tabel 1. Formation of 9a-g using pressurized microwave and conventional heating procedures

\begin{tabular}{ccccc}
\hline $\begin{array}{c}\text { Compd. } \\
\text { no. }\end{array}$ & \multicolumn{2}{c}{ Microwave Irradiation } & \multicolumn{2}{c}{ Conventional Heating } \\
\cline { 2 - 5 } & Time (min) & Yield (\%) & Time (h) & Yield (\%) \\
\hline 9a & 10 & 93 & 20 & 76 \\
9b & 7 & 90 & 21 & 74 \\
9c & 6 & 85 & 24 & 70 \\
9d & 6 & 90 & 22 & 72 \\
$9 \mathbf{9}$ & 8 & 83 & 20 & 74 \\
99 & 8 & 88 & 20 & 72 \\
99 & 10 & 82 & 24 & 70 \\
\hline
\end{tabular}

$\alpha$-Haloketones and $\alpha$-haloesters have demonstrated synthetic application for construction of heterocyclic azoles via their reactions with bis-nucleophiles. ${ }^{8}$ In continuation of these studies, we examined the reactions of 3-thioxo-2,3,4,7,8,9-hexahydro-1H-benzo[6',7']cyclohepta[1',2':4,5]pyrido[2,3- $d]$ pyrimidin-1-one (3) with $\alpha$-haloketones and $\alpha$-haloester in acetic acid in the presence of sodium acetate under various conditions (conventional heating and pressurized microwave irradiation). In both cases, the corresponding novel penta-heterocyclic 6,7-dihydrobenzo[6',7']cyclohepta[1',2':4,5]pyrido[2,3- $d][1,3]$ thiazolo[3,2-a] pyrimidin-15(5H)ones 11a-d were isolated as shown in Scheme 3 (Table 2). The structure of the isolated products was established on the basis of IR, ${ }^{1} \mathrm{H}$ NMR, mass spectra and elemental analyses (see Exprimental Section).

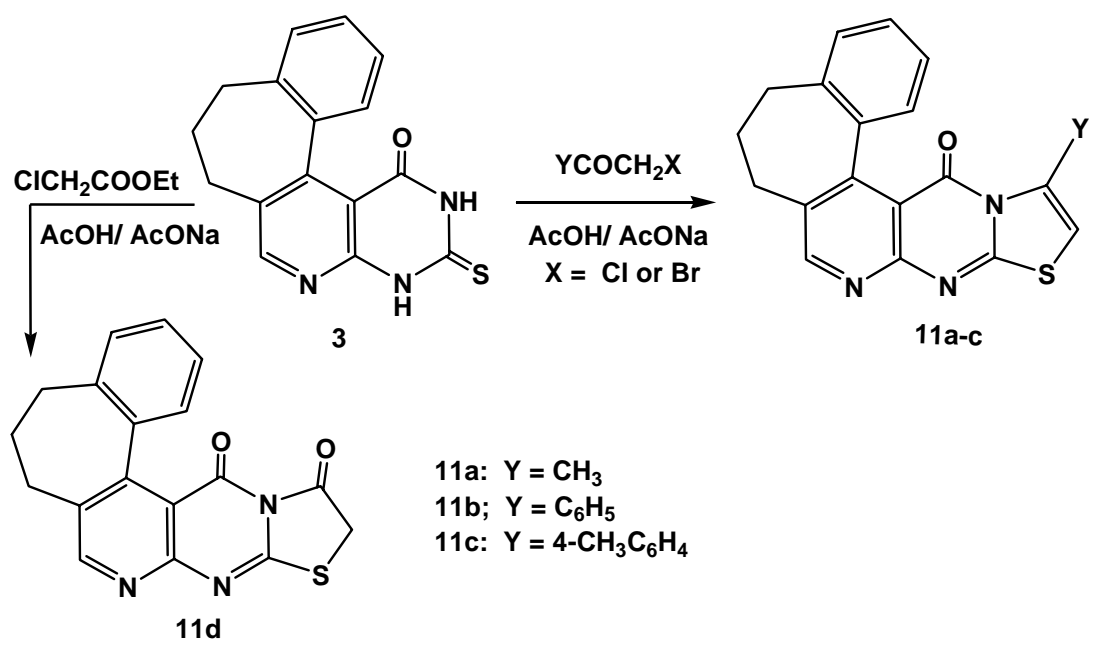




\section{Scheme 3}

Tabel 2. Formation of 11a-d using pressurized microwave and conventional heating procedures

\begin{tabular}{ccccc}
\hline \multirow{2}{*}{$\begin{array}{c}\text { Compd. } \\
\text { no. }\end{array}$} & \multicolumn{2}{c}{ Microwave Irradiation } & \multicolumn{2}{c}{ Conventional Heating } \\
\cline { 2 - 5 } Time (min) & Yield (\%) & Time (h) & Yield (\%) \\
\hline 11a & 8 & 90 & 4 & 72 \\
11b & 7 & 88 & 3 & 70 \\
11c & 8 & 91 & 4 & 73 \\
11d & 8 & 90 & 4 & 68 \\
\hline
\end{tabular}

\section{Conclusions}

We described in this context a synthetic route for new penta-heterocyclic ring systems from hydrazonoyl halides and tetra-heterocyclic thione in one-pot reactions. The microwave-assisted process, in contrast to conventional heating, gave the desired products in higher yields with shorter reaction times. In pressurized microwave, the solvent can be heated up to temperatures from 2 to 4 times their respective boiling points which enhanced the reaction rates.

\section{Experimental Section}

General Procedures. All melting points were determined on an electrothermal Gallenkamp apparatus and are uncorrected. Solvents were generally distilled and dried by standard literature procedures prior to use. The IR spectra were measured on a Pye-Unicam SP300 instrument in potassium bromide discs. The ${ }^{1} \mathrm{H}$ and ${ }^{13} \mathrm{C}-\mathrm{NMR}$ spectra were recorded on a Varian Mercury VXR-300 spectrometer (300 MHz for ${ }^{1} \mathrm{H}-\mathrm{NMR}$ and $75 \mathrm{MHz}$ for ${ }^{13} \mathrm{C} \mathrm{NMR}$ ) and the chemical shifts were related to that of the solvent DMSO- $\mathrm{d}_{6}$. The mass spectra were recorded on a GCMSQ1000-EX Shimadzu and GCMS 5988-A HP spectrometers, the ionizing voltage was $70 \mathrm{eV}$. Elemental analyses were carried out by the Microanalytical Center of Cairo University, Giza, Egypt. Microwave experiments were carried out using CEM Discover Labmate microwave apparatus (300 W with Chem. Driver Software). Hydrazonoyl halides 6a-g were prepared following literature methods. ${ }^{26-31}$

General procedures for the synthesis of 3-thioxo-2,3,4,7,8,9-hexahydro-1H-benzo [6',7']cyclohepta[1',2':4,5]pyrido[2,3-d]pyrimidin-1-one (3) 
Method A. A mixture of enaminone $(\mathbf{1})^{32}(2.15 \mathrm{~g}, 10 \mathrm{mmol})$ and 6-amino-2-thioxo-2,3dihydropyrimidin-4(1H)-one (2) $(1.43 \mathrm{~g}, 10 \mathrm{mmol})$ in glacial acetic acid $(30 \mathrm{~mL})$ was heated under reflux for $5 \mathrm{~h}$. or under pressurized microwave irradiation for $5 \mathrm{~min}$. After cooling, the reaction mixture was poured into $\mathrm{ice} / \mathrm{HCl}$ mixture and the solid product was collected and recrystallized from DMSO as pale yellow crystals [78\% thermal, 92\% Microwave], mp $278{ }^{\circ} \mathrm{C}$ [Lit. mp $\left.275^{\circ} \mathrm{C}\right]^{21}$; IR $(\mathrm{KBr}) v=3360,3102(2 \mathrm{NH}), 1688(\mathrm{C}=\mathrm{O}) \mathrm{cm}^{-1} ;{ }^{1} \mathrm{H}$ NMR (DMSO-d 6$) \delta=$ 2.14-2.52 (m, 6H, 3CH $), 7.34-7.65(\mathrm{~m}, 4 \mathrm{H}, \mathrm{Ar}-\mathrm{H}), 8.23(\mathrm{~s}, 1 \mathrm{H}$, pyridine-H), $12.53(\mathrm{~s}, 1 \mathrm{H}, \mathrm{NH})$, 13.10 (s, 1H, NH) ppm; MS, m/z (\%) $297\left(\mathrm{M}^{+}+2,7\right), 296\left(\mathrm{M}^{+}+1,23\right), 295\left(\mathrm{M}^{+}, 100\right), 294$ (18), 252 (11), 207 (9), 153 (8), 103 (9), 77 (7),76 (8). Anal. Calcd. for $\mathrm{C}_{16} \mathrm{H}_{13} \mathrm{~N}_{3} \mathrm{OS}$ (295.08): C, 65.06; H, 4.44; N, 14.23; S, 10.86. Found: C, 65.11; H, 4.64; N, 14.42; S, 10.72\%.

Method B. A mixture of 6-amino-2-thioxo-2,3-dihydropyrimidin-4(1H)-one (2) ${ }^{33}(1.47 \mathrm{~g}, 10$ mmol) and dimethylformamide dimethylacetal (DMF-DMA) $(5 \mathrm{~mL})$ was refluxed for 3 hours. Methanol was added to the reaction mixture and the solid product was collected by filtration to give $N, N$-dimethyl- $N$-(6-oxo-2-thioxo-1,2,3,6-tetrahydropyrimidin-4-yl)imido- formamide (5). ${ }^{17}$ Refluxing of 5 (1.98 g, $10 \mathrm{mmol})$ with benzosuberone (1.6 g, $10 \mathrm{mmol})$ in acetic acid (30 $\mathrm{mL})$ for 5 hours yield the authentic product 3 , identical in all respects ( $\mathrm{mp}$, mixed $\mathrm{mp}$, IR) to that prepared by method A.

\section{General Procedure for the reaction of hydrazonoyl halides with 3-thioxo-2,3, 4,7,8,9- hexahydro-1H-benzo[6',7']cyclohepta[1',2':4,5]pyrido[2,3- $d]$ pyrimidin-1-one (3). Pressurized microwave method (method A)}

To a solution of $\mathbf{3}$ and the appropriate hydrazonoyl halides 6a-g (1 mmol of each) in dioxane (20 $\mathrm{mL})$ was added triethylamine $(0.14 \mathrm{~mL}, 1 \mathrm{mmol})$ at room temperature. The reaction mixture was irradiated in a pressurized microwave $\left(17.2 \mathrm{Bar}, 140{ }^{\circ} \mathrm{C}\right)$ for $6-10 \mathrm{~min}$. at a power of $300 \mathrm{~W}$. After cooling to room temperature, the solution was extracted with chloroform $(3 \mathrm{X} 10 \mathrm{ml})$. The organic extracts were dried over anhydrous magnesium sulfate, and then evaporated under reduced pressure. The solid residue was recrystallized from the appropriate solvent to give products 9a-g. Thermal method (method B). To a solution of $\mathbf{3}$ and the appropriate hydrazonoyl halides 6a-g $(1 \mathrm{mmol}$ of each) in dioxane $(20 \mathrm{~mL})$ was added triethylamine $(0.14 \mathrm{~mL}, 1 \mathrm{mmol})$. The reaction mixture was refluxed till all of the starting materials had disappeared (20-24 h, monitored by TLC). The solvent was evaporated and the residue was triturated with methanol. The solid formed was collected and recrystallized from the appropriate solvent to give products identical to those produced by microwave method.

\section{1,13-Diphenyl-5,6,7,11-tetrahydro-15H-benzo[6',7']cyclohepta[1',2':4,5]pyrido[2,3-d]} [1,2,4]triazolo[4,3-a]pyrimidin-15-one (9a). Pale yellow crystals (Ethyl acetate/petroleum ether), mp $296{ }^{\circ} \mathrm{C}$; IR (KBr) $v=1699(\mathrm{C}=\mathrm{O}) \mathrm{cm}^{-1} ;{ }^{1} \mathrm{H}$ NMR $\left(\mathrm{DMSO}-\mathrm{d}_{6}\right) \delta=2.17-2.59(\mathrm{~m}, 6 \mathrm{H}$, $\left.3 \mathrm{CH}_{2}\right), 7.36-8.35(\mathrm{~m}, 14 \mathrm{H}, \mathrm{Ar}-\mathrm{H}), 8.42\left(\mathrm{~s}, 1 \mathrm{H}\right.$, pyridine-H) ppm; ${ }^{13} \mathrm{C}$ NMR (DMSO-d $\left.{ }_{6}\right) \delta=$ 28.66, 31.59, 31.98, 109.91, 121.34, 125.96, 126.88, 129.01, 129.11, 129.34, 129.59, 130.51, 
132.62, 134.92, 136.05, 136.61, 137.21, 138.67, 139.24, 139.44, 143.62, 153.41, 155.36, 159.84, 163.21; MS, m/z (\%) 455 (M+, 100), 394 (61), 285 (33), 194 (28), 167 (50), 105 (17), 91 (61), 89 (56), 77 (60). Anal. Calcd. for $\mathrm{C}_{29} \mathrm{H}_{21} \mathrm{~N}_{5} \mathrm{O}$ (455.17): C, 76.47; H, 4.65; N, 15.37. Found: C, 76.31; H, 4.54; N, 15.42\%.

Ethyl 15-oxo-11-phenyl-6,7,11,15-tetrahydro-5H-benzo[6',7']cyclohepta[1',2':4,5] pyrido [2,3-d][1,2,4]triazolo[4,3-a]pyrimidine-13-carboxylate (9b). Pale yellow crystals (EtOH), mp $212{ }^{\circ} \mathrm{C}$; IR $(\mathrm{KBr}) \mathrm{v}=1749,1713(2 \mathrm{C}=\mathrm{O}) \mathrm{cm}^{-1} ;{ }^{1} \mathrm{H}$ NMR $\left(\mathrm{DMSO}-\mathrm{d}_{6}\right) \delta=1.53(\mathrm{t}, \mathrm{J}=7 \mathrm{~Hz}, 3 \mathrm{H}$, $\mathrm{CH}_{3}$ ), 1.55-2.67 (m, 6H, 3CH 2$), 4.63$ (q, $\left.\mathrm{J}=7 \mathrm{~Hz}, 2 \mathrm{H}, \mathrm{CH}_{2}\right), 7.27-7.94(\mathrm{~m}, 9 \mathrm{H}, \mathrm{Ar}-\mathrm{H}), 8.25$ (s, $1 \mathrm{H}$, pyridine-H) ppm; ${ }^{13} \mathrm{C}$ NMR $\left(\mathrm{DMSO}_{-} \mathrm{d}_{6}\right) \delta=17.05,28.82,30.22,31.99,66.31,110.40$, $112.11,120.68,126.51,128.67,128.83,129.32$, 129.89, 131.68, 135.90, 138.11, 139.43, 150.26, 159.67, 163.47, 166.05, 175.55, 179.80, 180.00; MS, $m / z(\%) 451\left(\mathrm{M}^{+}, 44\right), 378$ (50), 295 (56), 207 (26), 205 (20), 191 (17), 127 (22), 118 (30), 115 (28), 105 (13), 103 (30), 91 (46), 77 (100). Anal. Calcd. for $\mathrm{C}_{26} \mathrm{H}_{21} \mathrm{~N}_{5} \mathrm{O}_{3}$ (451.16): C, 69.17; H, 4.69; N, 15.51. Found: C, 69.31; H, 4.54; N, $15.41 \%$.

13-Acetyl-11-phenyl-5,6,7,11-tetrahydro-15H-benzo[6',7']cyclohepta[1',2':4,5]pyrido[2,3- $d$ ] $[1,2,4]$ triazolo[4,3-a]pyrimidin-15-one (9c). Yellow crystals $(\mathrm{EtOH}), \mathrm{mp} 230{ }^{\circ} \mathrm{C}$; $\mathrm{IR}(\mathrm{KBr}) \mathrm{v}=$ 1726, $1702(2 \mathrm{C}=\mathrm{O}) \mathrm{cm}^{-1} ;{ }^{1} \mathrm{H}$ NMR $\left(\mathrm{DMSO}_{6}\right) \delta=1.92-2.63\left(\mathrm{~m}, 6 \mathrm{H}, 3 \mathrm{CH}_{2}\right), 2.78(\mathrm{~s}, 3 \mathrm{H}$, $\left.\mathrm{COCH}_{3}\right), 7.35-8.27$ (m, 9H, Ar-H), 8.49 (s, 1H, pyridine-H) ppm; MS, $m / z(\%) 422\left(\mathrm{M}^{+}+1,30\right)$, 421 (M , 100), 378 (17), 207 (19), 192 (15), 77 (80). Anal. Calcd. for $\mathrm{C}_{25} \mathrm{H}_{19} \mathrm{~N}_{5} \mathrm{O}_{2}$ (421.15): C, 71.25; H, 4.54; N, 16.62. Found: C, 71.51; H, 4.34; N, 16.42\%.

15-Oxo- $N, 11$-diphenyl-6,7,11,15-tetrahydro-5H-benzo[6',7']cyclohepta[1',2':4,5]pyrido[2,3d] [1,2,4]triazolo[4,3-a]pyrimidine-13-carboxamide (9d). Yellow crystals (Dioxan/MeOH), mp $306{ }^{\circ} \mathrm{C}$; IR $(\mathrm{KBr}) v=3201(\mathrm{NH}), 1711,1672(2 \mathrm{C}=\mathrm{O}) \mathrm{cm}^{-1} ;{ }^{1} \mathrm{H}$ NMR $\left(\mathrm{DMSO}-\mathrm{d}_{6}\right) \delta=2.19-2.60$ $\left(\mathrm{m}, 6 \mathrm{H}, 3 \mathrm{CH}_{2}\right), 7.22-8.17(\mathrm{~m}, 14 \mathrm{H}, \mathrm{Ar}-\mathrm{H}), 8.49$ (s, $1 \mathrm{H}$, pyridine-H), $11.15(\mathrm{~s}, 1 \mathrm{H}, \mathrm{NH}) \mathrm{ppm} ;{ }^{13} \mathrm{C}$ NMR (DMSO-d $\left.{ }_{6}\right) \delta=28.76,31.68,32.39,110.31,120.97,126.07,127.62,128.48,128.58$, $128.90,129.58,130.61,131.62,133.42,135.65,136.64,137.43,138.20,139.13,139.74,145.62$, 153.45, 156.14, 159.70, 166.44, 179.51; MS, m/z (\%) $498\left(\mathrm{M}^{+}, 100\right), 484$ (32), 392 (27), 378 (25), 192 (34), 153 (28), 152(30), 128 (22), 120 (19), 119 (43), 117 (20), 104 (34), 91 (95), 77 (66). Anal. Calcd. for $\mathrm{C}_{30} \mathrm{H}_{22} \mathrm{~N}_{6} \mathrm{O}_{2}$ (498.18): C, 72.28; H, 4.45; N, 16.86. Found: C, 72.50; H, 4.64; N, 16.65\%.

13-Benzoyl-11-phenyl-5,6,7,11-tetrahydro-15H-benzo[6',7']cyclohepta[1',2':4,5]pyrido[2,3d] $[1,2,4]$ triazolo[4,3-a]pyrimidin-15-one (9e). Yellow crystals $(\mathrm{MeOH}), \mathrm{mp} 270{ }^{\circ} \mathrm{C}$; $\mathrm{IR}(\mathrm{KBr}) v$ $=1702,1688(2 \mathrm{C}=\mathrm{O}) \mathrm{cm}^{-1} ;{ }^{1} \mathrm{H}$ NMR $\left(\mathrm{DMSO}_{-} \mathrm{d}_{6}\right) \delta=2.18-2.58\left(\mathrm{~m}, 6 \mathrm{H}, 3 \mathrm{CH}_{2}\right), 7.36-8.31(\mathrm{~m}$, $14 \mathrm{H}, \mathrm{Ar}-\mathrm{H}), 8.41$ (s, 1H, pyridine-H) ppm; ${ }^{13} \mathrm{C}$ NMR $\left(\mathrm{DMSO}_{\mathrm{d}}\right) \delta=28.83,30.28,31.70$, 109.06, 120.40, 126.03, 127.05, 128.61, 128.97, 129.04, 129.29, 129.87, 129.97, 130.14, 131.68, $134.88,135.24,136.64,138.81,139.17,139.43,146.30,155.95,157.69,165.78,181.93$; MS, m/z (\%) $484\left(\mathrm{M}^{+}+1,13\right), 483\left(\mathrm{M}^{+}, 40\right), 378$ (20), 220 (10), 105 (93), 77 (100). Anal. Calcd. for $\mathrm{C}_{30} \mathrm{H}_{21} \mathrm{~N}_{5} \mathrm{O}_{2}$ (483.17): C, 74.52; H, 4.38; N, 14.48. Found: C, 74.31; H, 4.51; N, 14.40\%. 


\section{1-(4-Chlorophenyl)-13-(2-thienylcarbonyl)-5,6,7,11-tetrahydro-15H-benzo[6',7']} cyclohepta[1',2':4,5]pyrido[2,3- $d][1,2,4]$ triazolo[4,3-a]pyrimidin-15-one (9f). Pale orange crystals $(\mathrm{EtOH}), \mathrm{mp} 294{ }^{\circ} \mathrm{C}$; IR $(\mathrm{KBr}) v=1716,1694(2 \mathrm{C}=\mathrm{O}) \mathrm{cm}^{-1} ;{ }^{1} \mathrm{H}$ NMR $\left(\mathrm{DMSO}-\mathrm{d}_{6}\right) \delta=$ 2.19-2.59 (m, 6H, 3CH $), 7.23-7.53(\mathrm{~m}, 7 \mathrm{H}, \mathrm{Ar}-\mathrm{H}), 7.72$ (d, $J=8 \mathrm{~Hz}, 2 \mathrm{H}, \mathrm{Ar}-\mathrm{H}), 8.19$ (d, $J=8$ $\mathrm{Hz}, 2 \mathrm{H}, \mathrm{Ar}-\mathrm{H}), 8.44$ (s, 1H, pyridine-H) ppm; MS, $m / z(\%) 525\left(\mathrm{M}^{+}+2,7\right), 524\left(\mathrm{M}^{+}+1,8\right), 523$ $\left(\mathrm{M}^{+}, 15\right), 428$ (50), 111 (100). Anal. Calcd. for $\mathrm{C}_{28} \mathrm{H}_{18} \mathrm{ClN}_{5} \mathrm{O}_{2} \mathrm{~S}$ (523.09): C, 64.18; H, 3.46; N, 13.37; S, 6.12. Found: C, 64.32; H, 3.53; N, 13.40; S, 5.99\%.

\section{1-(4-Methylphenyl)-13-(1-naphthoyl)-5,6,7,11-tetrahydro-15H-benzo[6',7']cyclohepta} $[1 ', 2 ': 4,5]$ pyrido[2,3- $d][1,2,4]$ triazolo[4,3-a]pyrimidin-15-one (9g). Pale orange crystals $(\mathrm{EtOH}), \mathrm{mp} 262{ }^{\circ} \mathrm{C}$; IR $(\mathrm{KBr}) v=1700,1680(2 \mathrm{C}=\mathrm{O}) \mathrm{cm}^{-1} ;{ }^{1} \mathrm{H}$ NMR $\left(\mathrm{DMSO}_{-} \mathrm{d}_{6}\right) \delta=2.25-2.72$ $\left(\mathrm{m}, 6 \mathrm{H}, 3 \mathrm{CH}_{2}\right), 2.64$ (s, 3H, Ar- $\left.\mathrm{CH}_{3}\right), 7.31$ (d, $\left.J=7 \mathrm{~Hz}, 2 \mathrm{H}, \mathrm{Ar}-\mathrm{H}\right), 7.42-7.99$ (m, 11H, Ar-H), 8.00 (d, $J=7 \mathrm{~Hz}, 2 \mathrm{H}, \mathrm{Ar}-\mathrm{H}), 8.18$ (s, 1H, pyridine-H) ppm; MS, $m / z$ (\%) $547\left(\mathrm{M}^{+}, 100\right), 392$ (50),180 (10), 158 (3), 128 (14), 106 (24), 91 (16), 77 (37). Anal. Calcd. for $\mathrm{C}_{35} \mathrm{H}_{25} \mathrm{~N}_{5} \mathrm{O}_{2}$ (547.20): C, 76.77; H, 4.60; N, 12.79. Found: C, 76.62; H, 4.53; N, 12.60\%.

Alternative synthesis of 11,13-diphenyl-5,6,7,11-tetrahydro-15H-benzo[6',7']cyclohepta [1',2':4,5]pyrido[2,3- $d][1,2,4]$ triazolo[4,3-a]pyrimidin-15-one (9a). A mixture of 7-amino-1,3diphenyl[1,2,4]triazolo[4,3-a]pyrimidin-5(1H)-one $(\mathbf{1 0})^{25}(0.303 \mathrm{~g}, 1 \mathrm{mmol})$ and enaminone (1) $(0.215 \mathrm{~g}, 1 \mathrm{mmol})$ in acetic acid was refluxed for 6 hours. After cooling, the mixture was poured into ice and the solid product was filtered off and recrystallized from (ethyl acetate/ petroleum ether) mixture to give compound 9a.

General procedure for the reaction of $\alpha$-haloketones or $\alpha$-haloester with 3-thioxo2,3,4,7,8,9-hexahydro-1H-benzo[6',7']cyclohepta[1',2':4,5]pyrido[2,3-d]pyrimidin-1-one (3) Pressurized microwave method $(\operatorname{method} A)$

To a solution of $3(0.295 \mathrm{~g}, 1 \mathrm{mmol})$ in acetic acid $(20 \mathrm{~mL})$ in the presence of sodium acetate $(0.082 \mathrm{~g}, 1 \mathrm{mmol})$ was added the appropriate $\alpha$-haloketones or $\alpha$-haloester $(1 \mathrm{mmol}$ of each) at room temperature. The reaction mixture was irradiated in a pressurized microwave (15.2 Bar, 120 ${ }^{\circ} \mathrm{C}$ ) for 7-8 min. at a power of $300 \mathrm{~W}$. After cooling to room temperature, the solution was extracted with chloroform $(3 \times 10 \mathrm{~mL})$. The organic extracts were dried over anhydrous magnesium sulfate, and evaporated under reduced pressure. The solid residue was recrystallized from ethanol to give products 11a-d.

Thermal method (method B). To a solution of $3(0.295 \mathrm{~g}, 1 \mathrm{mmol})$ in acetic acid $(20 \mathrm{~mL})$ in the presence of sodium acetate $(0.082 \mathrm{~g}, 1 \mathrm{mmol})$ was added the appropriate $\alpha$-haloketones or $\alpha$ haloester ( $1 \mathrm{mmol}$ of each) at room temperature. The reaction mixture was refluxed till all of the starting materials had disappeared (3-4 h, monitored by TLC). The solvent was evaporated and the residue was triturated with methanol. The solid formed was collected and recrystallized from ethanol to give products identical to those obtained by microwave method. 
13-Methyl-6,7-dihydrobenzo[6',7']cyclohepta[1',2':4,5]pyrido[2,3-d][1,3]thiazolo [3,2-a] pyrimidin-15(5H)-one (11a). Pale yellow crystals, mp $302{ }^{\circ} \mathrm{C}$; IR $(\mathrm{KBr}) v=1682(\mathrm{C}=\mathrm{O}) \mathrm{cm}^{-1}$; ${ }^{1} \mathrm{H}$ NMR $\left(\right.$ DMSO-d $\left._{6}\right) \delta=2.52\left(\mathrm{~s}, 3 \mathrm{H}, \mathrm{CH}_{3}\right), 2.54-2.67\left(\mathrm{~m}, 6 \mathrm{H}, 3 \mathrm{CH}_{2}\right), 7.27-7.84(\mathrm{~m}, 4 \mathrm{H}, \mathrm{Ar}-\mathrm{H})$, 7.91 (s, 1H, thiazole-H), 8.22 (s, 1H, pyridine-H) ppm; MS, m/z (\%) $333\left(\mathrm{M}^{+}, 100\right), 318(50)$, 292 (60), 215 (30), 143 (25), 118 (10), 76 (20). Anal. Calcd. for $\mathrm{C}_{19} \mathrm{H}_{15} \mathrm{~N}_{3} \mathrm{OS}$ (333.09): C, 68.45; H, 4.53; N, 12.60; S, 9.62. Found: C, 68.31; H, 4.44; N, 12.41; S, 9.52\%.

13-Phenyl-6,7-dihydrobenzo[6',7']cyclohepta[1',2':4,5]pyrido[2,3- $d][1,3]$ thiazolo [3,2-a] pyrimidin-15(5H)-one (11b). Orange crystals, mp $196{ }^{\circ} \mathrm{C}$; $\mathrm{IR}(\mathrm{KBr}) v=1685(\mathrm{C}=\mathrm{O}) \mathrm{cm}^{-1} ;{ }^{1} \mathrm{H}$ NMR $\left(\right.$ DMSO-d $\left._{6}\right) \delta=2.48-2.69\left(\mathrm{~m}, 6 \mathrm{H}, 3 \mathrm{CH}_{2}\right), 7.29-7.85(\mathrm{~m}, 9 \mathrm{H}, \mathrm{Ar}-\mathrm{H}), 7.78(\mathrm{~s}, 1 \mathrm{H}$, thiazoleH), 8.24 (s, 1H, pyridine-H) ppm; MS, m/z (\%) 395 (M+100), 292 (40), 143 (30), 118 (25), 102 (25), 91 (10), 77 (60). Anal. Calcd. for $\mathrm{C}_{24} \mathrm{H}_{17} \mathrm{~N}_{3} \mathrm{OS}$ (395.11): C, 72.89; H, 4.33; N, 10.63; S, 8.11. Found: C, 72.81; H, 4.37; N, 10.45; S, 8.19\%.

13-(4-Methylphenyl)-6,7-dihydrobenzo[6',7']cyclohepta[1',2':4,5]pyrido[2,3-d] [1,3]thiazolo [3,2-a]pyrimidin-15(5H)-one (11c). Orange crystals, mp $186{ }^{\circ} \mathrm{C}$; IR $(\mathrm{KBr}) v=1680(\mathrm{C}=\mathrm{O}) \mathrm{cm}^{-}$ ${ }^{1} ;{ }^{1} \mathrm{H}$ NMR $\left(\mathrm{DMSO}_{-} \mathrm{d}_{6}\right) \delta=2.42\left(\mathrm{~s}, 3 \mathrm{H}, \mathrm{Ar}-\mathrm{CH}_{3}\right), 2.44-2.67\left(\mathrm{~m}, 6 \mathrm{H}, 3 \mathrm{CH}_{2}\right), 7.27-7.30$ and 7.427.75 (m, 4H, Ar-H), 7.36 (d, $J=7 \mathrm{~Hz}, 2 \mathrm{H}, \mathrm{Ar}-\mathrm{H}), 7.81$ (d, $J=7 \mathrm{~Hz}, 2 \mathrm{H}, \mathrm{Ar}-\mathrm{H}), 7.98$ (s, 1H, thiazole-H), 8.21 (s, 1H, pyridine-H) ppm; MS, m/z (\%) $409\left(\mathrm{M}^{+}, 60\right), 291$ (100), 143 (25), 118 (15), 91 (60), 77 (40). Anal. Calcd. for $\mathrm{C}_{25} \mathrm{H}_{19} \mathrm{~N}_{3} \mathrm{OS}$ (409.12): C, 73.32; H, 4.68; N, 10.26; S, 7.83. Found: C, 73.21; H, 4.47; N, 10.40; S, 7.59\%.

6,7-Dihydrobenzo[6',7']cyclohepta[1',2':4,5]pyrido[2,3-d][1,3]thiazolo[3,2-a] pyrimidine13,15(5H,12H)-dione (11d). Pale yellow crystals, mp $290{ }^{\circ} \mathrm{C}$; IR (KBr) v = 1699, $1680(\mathrm{C}=\mathrm{O})$ $\mathrm{cm}^{-1} ;{ }^{1} \mathrm{H}$ NMR $\left(\mathrm{DMSO}_{6}\right) \delta=2.44-2.67\left(\mathrm{~m}, 6 \mathrm{H}, 3 \mathrm{CH}_{2}\right), 4.38\left(\mathrm{~s}, 2 \mathrm{H}\right.$, thiazole- $\left.\mathrm{CH}_{2}\right), 7.27-7.64$ $(\mathrm{m}, 4 \mathrm{H}, \mathrm{Ar}-\mathrm{H}), 8.27$ (s, 1H, pyridine-H) ppm; ${ }^{13} \mathrm{C}$ NMR $\left(\right.$ DMSO-d $\left._{6}\right) \delta=28.62,30.46,32.19$, $44.56,119.26,127.35,128.21,128.93,129.14,136.45,138.26,139.41,150.10,151.61,155.42$, 159.76, 162.60, 169.20; MS, m/z (\%) $335\left(\mathrm{M}^{+}, 40\right), 293$ (100), 217 (20), 143 (30), 118 (25), 76 (30). Anal. Calcd. for $\mathrm{C}_{18} \mathrm{H}_{13} \mathrm{~N}_{3} \mathrm{O}_{2} \mathrm{~S}$ (335.07): C, 64.46; H, 3.91; N, 12.53; S, 9.56. Found: C, 64.21; H, 4.07; N, 12.43; S, 9.69\%.

\section{References}

1. Robl, J. A.; Bang-Chi, C.; Chong-Qing, S. US Patent US 20002061 901; Chem. Abstr. 2001, 136, 401651.

2. Ganguly, A. K.; Doll, A. K.; Girijavallabhan, V. M. Curr. Med. Chem. 2001, 8, 1419.

3. Irene Giorgi, I.; Bianucci, A. M.; Biagi, G.; Livi, O.; Scartoni, V.; Leonardi, M.; Pietra, D.; Coi, A.; Massarelli, I.; Fatena, F. A.; Fiamingo, F. L.; Anastasi, P.; Giannini, G. Eur. J. Med. Chem. 2007, 42, 1.

4. Awal, K. Ger. Pat. 3839 711, 1989; Chem. Abstr. 1990, 112, 55902. 
5. Barthelmy, G.; Hallot, A.; Vallat, J. N. Fr. Pat. 2549 834, 1985; Chem. Abstr. 1985, 103, 71335u.

6. Bru-Magniez, N.; Guengor, T.; Teulon, J. M. US Pat. 5387 747, 1995; Chem. Abstr. 1995, $123,228204 \mathrm{p}$.

7. Nakamura, H.; Hosor, Y.; Fukacoa, J. Jpn. Kokai 0313 934, 1991; Chem. Abstr., 1991, 115, $60769 \mathrm{k}$.

8. Zhi, H.; Chen, L.; Zhang, L.; Liu, S.; Wan, D. C. C.; Lin, H.; Hu, C. Arkivoc 2008, (xiii), 266.

9. Andrade, C. K. Z.; Barreto, A. S.; Silva, W. A. Arkivoc 2008, (xii), 226.

10. Lidstrom, P.; Tierney, J.; Wathey, B.; Westman, J. Tetrahedron 2001, 57, 9225.

11. Bortolini, O.; D'Agostino, M.; De Nino, A.; Maiuolo, L.; Nardi, M.; Sindona, G. Tetrahedron 2008, 64, 8078.

12. El Ashry, E. H.; Kassem, A. A. Arkivoc 2006, (ix), 1.

13. Riyadh, S. M.; Al-Matar, H. M. Org. Prep. Proced. Int. 2007, 39, 385.

14. Hassaneen, H. M.; Abdallah, T. A.; Abdelhadi, H. A., Hassaneen, H. M. E.; Pagni, R. M. Heteroatom Chem. 2003, 14, 491.

15. Hassaneen, H. M. E. Arkivoc 2007, (i), 154.

16. Quiroga, J.; Rengifo, A.; Insuasty, B.; Abonı', R.; Nogueras, M.; Sa'nchez, A. Tetrahedron Lett. 2002, 43, 9061.

17. Hassaneen, H. M.; Abdallah, T. A. Molecules 2003, 8, 333.

18. Riyadh, S. M.; Abdelhamid, I. A.; Al-Matar, H. M.; Helmy, N. M.; Elnagdi, M. H. Heterocycles 2008, 75, 1849.

19. Dawood, K. M. J. Heterocycl. Chem. 2005, 42, 221.

20. Al-Saleh, B.; El-Apasery, M. A.; Abdel-Aziz, R. S.; Elnagdi, M. H. J. Heterocycl. Chem. 2005, 42, 563.

21. Remp, W.; Junek, H. Monatsh. Chem. 1973, 104, 1101.

22. Shawali, A. S.; Farghaly, T. A. Arkivoc 2008, (i), 18.

23. Geis, A. A.; Kamal-Eldeen, A. M.; Abdelhafez, A. A.; Gaber, A. M. Phosphorus, Sulfur, Silicon Relat. Elem. 1991, 56, 87.

24. Elliott, A. J.; Callaghan, P. D.; Gibson, M. S.; Nemeth, S. T. Can. J. Chem. 1975, 53, 1484.

25. Mosselhi, M. A. N. Monatsh. Chem. 2002, 133, 1297.

26. Shawali, A. S.; Osman, A. Tetrahedron 1971, 27, 2517.

27. Shawali, A. S.; Eweiss, N. F.; Hassaneen, H. M.; Algharib, M. S. Bull. Chem. Soc. Jpn. 1973, 48, 365 .

28. Shawali, A. S.; Abdelhamid, A. O. Bull. Chem. Soc. Jpn. 1976, 49, 321.

29. Parkanyi, C.; Shawali, A. S. J. Heterocycl. Chem. 1980, 17, 897.

30. Eweiss, N. F.; Osman, A. J. Heterocycl. Chem. 1980, 17, 1713.

31. Wolkoff, P. Can. J. Chem. 1975, 53, 1333.

32. Farghaly, T. A.; Abdel-Hafez, N. A.; Ragab, E. A.; Awad, H. H. Submitted for publication. 
33. Hubsch, W.; Pfleiderer, W. Helv. Chem. Acta 1988, 71, 1379. 